\section{Infarction of the Corpus Callosum: A Manifestation of Giant Cell Arteritis}

Cerebral vasculitis is a known cause of ischemic and hemorrhagic strokes and has been described as one of the rare but important causes of corpus callosum infarction. Biopsy-proved giant cell arteritis causing callosal infarction is an exceedingly rare finding because a tissue specimen is usually not obtained and conclusions are drawn on the basis of clinical and radiologic findings alone. We present a case of callosal infarction, which evolved and eventually affected large portions of both cerebral hemispheres.

A 63-year-old woman presented to our hospital with left-sided numbness and neglect, cognitive changes, and apraxia. One month earlier, she was found to have a C-reactive protein level of $8.0 \mathrm{mg} / \mathrm{dL}$ (normal $<0.5 \mathrm{mg} / \mathrm{dL}$ ) and $75 \%$ stenosis in both femoral arteries. These results prompted a temporal artery biopsy, yielding a diagnosis of giant cell arteritis. On admission, head CT demonstrated low attenuation in the splenium of the corpus callosum (Fig 1). Considerations included infarction, tumor, demyelination, or infection. Subsequent CT angiography (CTA) revealed segmental narrowing and beading involving the petrous segment of both internal carotid arteries (Fig 2) and along the splenial or posterior pericallosal arteries within the posterior circulation. The condition of the patient continued to decline during the following week, culminating in a coma. An MR image obtained 12 days after the initial presentation revealed involvement of the entire corpus callosum, both cerebral hemispheres, and the right cerebellum with restricted diffusion indicating infarction secondary to intracranial vasculitis (Fig 3). In addition, hemorrhage was noted in the cortical gyri (Fig 4).

Lesions of the corpus callosum can present a diagnostic dilemma, both for the radiologist and the clinician. Clinically, they are associated with neuropsychiatric symptoms, mainly interhemispheric disconnection syndromes. Patients may experience gait disorders, apraxia, agraphia, and tactile anomia. ${ }^{1}$ While the corpus callosum is affected by varying disease processes, ischemia tends to spare this unique white matter structure. One accepted theory that explains relative callosal immunity to infarction relates to its rich blood supply delivered from the anterior and posterior circulations. In a study by Chrysikopoulos et al, ${ }^{2}$ the authors described the splenium as being most commonly involved in thromboembolic causes of callosal infarction, very likely due to the predilection for posterior cerebral artery versus anterior cerebral artery territory ischemia. In our patient, the lesion began in the splenium and progressed rostrally to involve

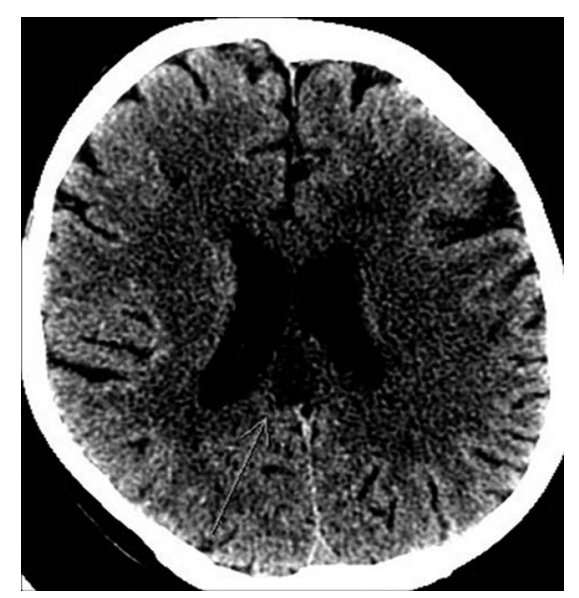

Fig 1. Noncontrast head CT scan demonstrates low attenuation in the splenium of the corpus callosum (arrow).

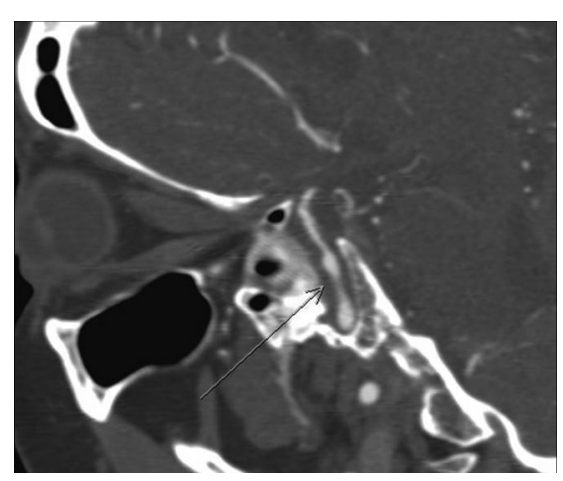

Fig 2. Oblique sagittal reformatted maximum intensity projection image from a CTA image depicts beading and narrowing of the left internal carotid artery (arrow).

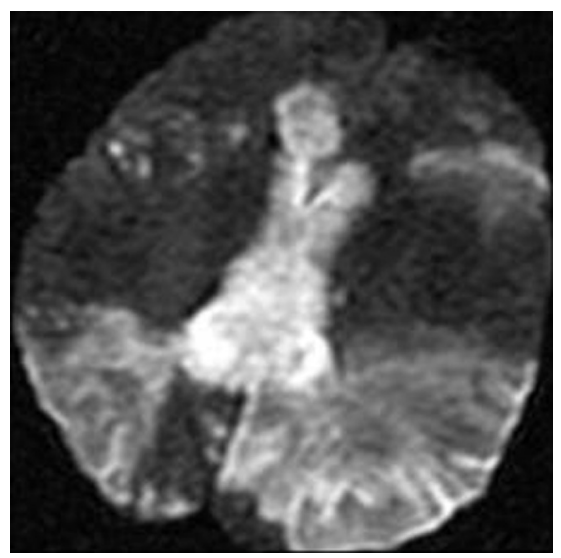

Fig 3. Diffusion-weighted image shows hyperintensity in the corpus callosum and within both cerebral hemispheres.

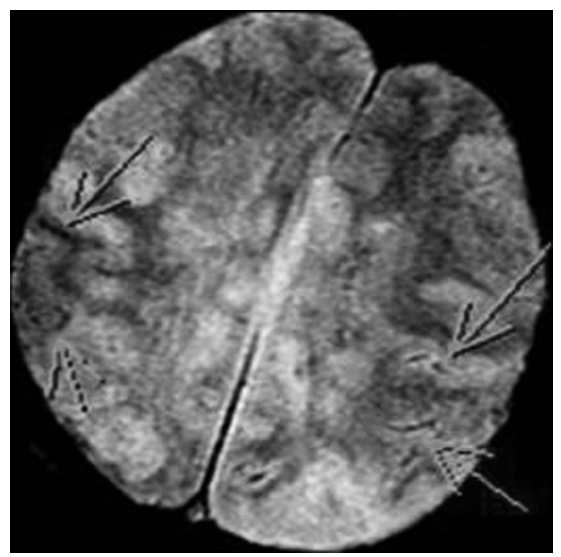

Fig 4. T2-weighted gradient recalled-echo image shows curvilinear areas of decreased signal intensity within the left parietal lobe, indicating susceptibility artifacts resulting from hemorrhage (arrows).

the body and genu and eventually a large portion of both cerebral hemispheres. The global involvement in our case was secondary to diffuse vasculitis rather than more focal thromboembolic disease.

Johnson et $\mathrm{al}^{3}$ described a case of biopsy-proved cerebral vasculitis that was seen as bilateral parieto-occipital "masses" that crossed the splenium of the corpus callosum. Greenan et $\mathrm{al}^{4}$ described a series of 7 clinically suspected cases of cerebral vasculitis in which only 1 was biopsy-proved arteritis, but that single case did not involve the corpus callosum. Other cases in that series did cause infarcts in the deep white matter, including the corpus callosum and capsular tracts, but their diagnosis of vasculitis was based on clinical and radiographic findings 
alone. We have thus presented an unusual case of corpus callosum infarction with a known causal entity of vasculitis.

J. Lempel

S. Bernstein

Norwalk Hospital

Yale University Medical School

Norwalk, Connecticut

P. Kalina

Mayo Clinic

Rochester, Minnesota

\section{References}

1. Giroud M, Dumas R. Clinical and topographical range of callosal infarction: a clinical and radiological correlation study. J Neurol Neurosurg Psychiatry 1995;59:238-42

2. Chrysikopoulos H, Andreou J, Roussakis A, et al. Infarction of the corpus callosum: computed tomography and magnetic resonance imaging. Eur $J$ Radiol 1997;25:2-8

3. Johnson M, Maciunas R, Dutt P, et al. Granulomatous angiitis masquerading as a mass lesion. Surg Neurol 1989;31:49-53

4. Greenan TJ, Grossman RI, Goldberg HI. Cerebral vasculitis: MR imaging and angiographic correlation. Radiology 1992;182:65-72

http://dx.doi.org/10.3174/ajnr.A2763 